\title{
Mechanical Properties of Nanocomposite
}

\author{
Mashal Alenazi \\ Department of Biomedical Engineering, University of Bridgeport, Bridgeport, CT, USA
}

\begin{abstract}
The effect of microstructure on thermal stability and mechanical properties of co-sputtered deposited $\mathrm{Zr}-\mathrm{W}-$ $\mathrm{B}-\mathrm{N}$ thin films on $\mathrm{Si}(100)$ substrates have been studied in detail. The power density of boron target has been varied from 0.1 to $7.5 \mathrm{~W} / \mathrm{cm} 2$ to obtained films of varying microstructure. It was found that there were a lot of dislocations and twins generated in the composite. The process of dislocation or twin generation can dissipate much fracture energy and then increase the fracture toughness.
\end{abstract}

Keywords: Mechanical, Nanocomposite, Ceramic, AL2O3/SIC.

\section{INTRODUCTION}

Nitride-based hard compound coatings were vogue in it was proved that the whiskers are better than the particles; 1980s because of their excellent properties such as high however, whiskers are not very practicable for the high hardness, wear resistance, thermal stability and corrosion price and the toxicity. With the development of the nanoresistance [1] and [2], but TiN coatings are not stable scale powder, a great breakthrough in ceramic beyond $550{ }^{\circ} \mathrm{C}$ and oxidize rapidly [3] and [4]. (Ti-Al)N nanocomposites has been achieved in the world. Almost coatings offer better suitability when oxidation resistance all the reported Al2O3/SiC nanocomposites were at higher temperature is required particularly in the higher fabricated with single micro-scale $\mathrm{Al} 2 \mathrm{O} 3$ as matrix with cutting speed range as compared to TiN coatings [5]. This an incorporation of single nano-scale SiC, and the results is because of the formation of Al-rich oxide layer at the showed the significant improvement in the flexural top most surface and inward diffusion of oxygen to form a strength while the modest increase in fracture toughness [3] Ti-rich oxide at the coating-substrate interface. This and [5].

double oxide layer has been found to reduce further oxidation by inhibiting oxygen diffusion to the underlying coating. For these reasons, TiAlN films are usually used as thermal barrier, contact materials in microelectronics, catalytic and decoration of surface and wear protection. Owing to their applications, investigations have been carried out on materials synthesis and their mechanical, physical, and chemical properties of TiAlN films. A crucial factor of many major industrial sectors, such as material processing, power generation, chemical engineering and aerospace, is an ability to operate in exotic environment. For tribological applications coatings exhibiting high thermal stability, wear resistance and fracture toughness are required. For these applications, nanocomposite nitride coatings can make a huge positive impact. Nanocomposite nitride coatings represent a new generation of materials [3], [4] and [5]. Because of their superior mechanical properties (in particular, superhardness and supertoughness) and high chemical inertness, these coatings can significantly lower friction, wear losses and at the same time increase resistance to fatigue and corrosion, which have increasingly become the life-limiting factors for mechanical components in many industrial applications [4] and [5]. Ceramic materials have intrinsic advantages, such as high melting point, high hardness, and good chemical stability at high temperature, but the low flexural strength and fracture toughness limit the further applications such as for the cutting tool. Therefore, many works have been developed for improving their mechanical properties, originally most researchers focused on adding the micro-scale particles or whiskers to the matrix so that the fracture toughness of the composite was highly improved [1] and [2], furthermore,

\section{Synthesis Al203/SiC}

There are different boundaries in $\mathrm{Al} 2 \mathrm{O} 3 / \mathrm{SiC} \mu / \mathrm{SiCn}$ nanocomposite; they are $\mathrm{Al} 2 \mathrm{O} 3 / \mathrm{Al} 2 \mathrm{O} 3$ grain boundary, $\mathrm{Al} 2 \mathrm{O} 3 / \mathrm{SiC}$ interface and sub-grain boundary which exist only when the $\mathrm{SiC}$ nanoparticle is located within the Al2O3 matrix grain. The mechanical properties and the fracture mode are dependent on the strength of grain boundary. In the present work, the designed microstructure for ASs nanocomposite is shown in Fig. 1(a); nano-scale $\mathrm{SiC}$ particles are located both within the alumina matrix grains and at the $\mathrm{Al} 2 \mathrm{O} 3 / \mathrm{Al} 2 \mathrm{O} 3$ grain boundary. Owing to the large applicability of Boron in increasing thermal stability, the effect of Boron incorporation on novel $\mathrm{Zr}-$ $\mathrm{W}-\mathrm{N}$ developed by our group has been studied. The developed $\mathrm{Zr}-\mathrm{W}-\mathrm{N}$ coatings showed remarkable combination of high hardness and wear resistance but exhibited low thermal stability. The effect of Boron incorporation on thermal stability and mechanical properties of $\mathrm{Zr}-\mathrm{W}-\mathrm{N}$ has been studied. Nanocomposite $\mathrm{Zr}-\mathrm{W}-\mathrm{B}-\mathrm{N}$ thin films deposited on $\mathrm{Si}$ (100) substrate have been investigated in detail as a function of varying deposition parameters to substantiate the influence of microstructures on its mechanical properties. In order to ensure compatibility and stability of the sputter deposited $\mathrm{Zr}-\mathrm{W}-\mathrm{B}-\mathrm{N}$ thin films in tribological applications, the present work has been focused to investigate the $\mathrm{DC} / \mathrm{RF}$ magnetron sputtered nanocomposite $\mathrm{Zr}-\mathrm{W}-\mathrm{B}-\mathrm{N}$ thin films with varying $\mathrm{B}$ content and hence varying microstructure. The films were characterized by XRD, FE-SEM, AFM, Raman and TEM to reveal the influence of processing parameters on microstructural characteristics and the atomic percentage of $\mathrm{B}$ in the $\mathrm{Zr}-\mathrm{W}-\mathrm{B}-\mathrm{N}$ thin films were 
measured using Wavelength Dispersive Spectroscopy (WDS) attached with EPMA.

Thermal stresses arise from thermal mismatch between coating and substrate during final cooling from deposition to room temperature, its effect becomes more exaggerated when multiple layers exist [19]. Intrinsic stresses in PVD coatings arise during deposition and its magnitude depends on deposition conditions, such as reference potential on substrate, pressure of working gas and target to substrate distance as well as on the stoichiometry and thickness of the coating layers reported that $(\mathrm{Til}-\mathrm{xAlx}) \mathrm{N}$ films present a face centered cubic structure (fcc), when $\mathrm{Al}$ concentration $(\mathrm{x})$ is less than 0.6 . These results also match well with the previous studies [23] and [24]. The tendency of broadening of the TiAlN (200) and MoN (202) was also observed indicating the diminution of the grain size or the residual stress induced in the crystal lattice. MoN peak is also observed at $36.0^{\circ}$, corresponding to expanded hexagonal MoN (PDF-89-5024), in agreement with the literature. The peak at $62^{\circ}$ is also identified as TiAlN (220). There exists elastic strain energy in the dislocations, which means the dislocation has a tendency to shorten so as to reduce the energy, i.e., a curving dislocation inclines to turn straight, dislocation loop inclines to shrink and even disappear. Thus, on one side, the elastic strain energy in dislocations can be consumed; on the other hand, some fracture energy can be absorbed by the dislocation deformation while the crack propagating to the dislocations, that is the pinning effect. Furthermore, when the dislocation density increases to a certain extent, the microcracks will be produced. Microcrack can also absorb some fracture energy by either making the main cracks branch off or reducing the propagating speed of cracks. Therefore, the fracture toughness of the composite can be improved.

\section{RESULTS AND DISCUSSION}

After the foundation of nanotube and nanowires, in recent years nanobelts or nanoribbons have been reported by professor Wang and co-workers, the nanobelt has a cross section of narrow rectangle, with the width of 30-300 nm and the thickness of 5-10 $\mathrm{nm}$, the length of nanobelt can reach to several millimeters. Compared with carbon nanotube, nanobelt has particularly predominant microstructure and physical properties. However, the study on nanobelt is still in its early stages, and was mainly focused on the synthesis and microstructure rather than on the mechanical properties.

Modification of C60 NR surface, through a photopolymerization process, we are able to stabilize the surface so that it can be homogeneously decorated by the palladium nanoparticles in a solution process, without any structural damage. This gives a material that exhibits a much more homogeneous and well-distributed decoration of Pd nanoparticles than reference samples of nonpolymerized C60 NRs.

Mn-doped ZnS NCs with good luminescence property could be synthesized with the similar method. Then, the hydroxyl-ending alkyl group thus introduced onto the surface of $\mathrm{ZnS}$ NCs enhances their dispersity in solvent, allowing the particle size of NCs to be controlled. We have found that the particle size of the $\mathrm{ZnS} \mathrm{NC}$ characterized by TEM is about $2.6 \mathrm{~nm}$, in agreement with the calculated data from UV-vis absorption spectra according to Brus's model and Debye-Scherrer formula. The surface morphology of the TiAlN/MoN multilayer coatings with different bilayer thickness is shown in Fig. $4 a-c$, a major concern for TiAlN/MoN multilayer coatings deposited by arc ion plating is the macro-particle (MP) formation, and this phenomenon is due to the evaporation of the metal macro-particles. In plasma environment there are three metal ions $\mathrm{Al}+, \mathrm{Ti}+$ and $\mathrm{Mo}+$ that react with nitrogen. It is believed that very fast evaporation during the cathodic arc process produces excess atoms that are not completely ionized before they arrive at the substrate surface. These excess neutral atoms may combine to form macro particles during the flight and other reason may be during arc evaporation process if cathode spot stays at an evaporative point for too long it can eject a large amount of macro-particles or droplets.

\section{CONCLUSION}

The advanced multi-scale ceramic nanocomposite $\mathrm{Al} 2 \mathrm{O} 3 / \mathrm{SiC} \mu / \mathrm{SiCn}$ was successfully fabricated; the strengthening and toughening mechanisms were deeply investigated according to the special microstructure observed with TEM and HRTEM. Prior to deposition all substrates were ultrasonically cleaned in acetone. Targets were fixed at an angle of $45^{\circ}$ to the substrate normal. Si substrates were mounted on the substrate holder held at negative potential with the help of silver paste. The substrate holder was rotated at $5 \mathrm{rpm}$ by using a DC motor to ensure uniformity in the deposited thin films. Meanwhile, the lattice of TiN insertion layer is forced to expand, and interfacial tensile stress forms. Therefore, the alternating compressive and tensile stress fields can be created along the growth direction. As the thickness of TiN insertion layer increases further, the epitaxial growth structure between TiSiN and TiN layers is subjected to damage.

\section{REFERNCES}

[1] Liu, H., Huang, C., Teng, X., \& Wang, H. (2008). Effect of special microstructure on the mechanical properties of nanocomposite. Materials Science and Engineering: A, 487(1-2), 258-263. doi:

2] Yousaf, M. I., Pelenovich, V. O., Yang, B., Liu, C. S., \& Fu, D. J. (2015). Effect of bilayer period on structural and mechanical properties of nanocomposite TiAlN/MoN multilayer films synthesized by cathodic arc ion-plating. Surface and Coatings Technology, 282, 94-102. doi:

[3] Haraguchi, K., Farnworth, R., Ohbayashi, A., \& Takehisa, T. (2003). Compositional effects on mechanical properties of nanocomposite hydrogels composed of poly (N, N-dimethylacrylamide) and clay. Macromolecules, 36(15), 5732-5741.

[4] Rafiee, M. A., Rafiee, J., Wang, Z., Song, H., Yu, Z. Z., \& Koratkar, N. (2009). Enhanced mechanical properties of nanocomposites at low graphene content. ACS nano, 3(12), 3884-3890.

[5] Agag, T., Koga, T., \& Takeichi, T. (2001). Studies on thermal and mechanical properties of polyimide-clay nanocomposites. Polymer, 42(8), 3399-3408 